\title{
Quantum Strings and Bethe Equations
}

\author{
G. Arutyunov*1 \\ ${ }^{1}$ Max-Planck-Institut für Gravitationsphysik, Albert-Einstein-Institut, \\ Am Mühlenberg 1, D-14476 Potsdam, Germany
}

Key words AdS-CFT Duality, Bethe Ansatz. PACS $11.25 \mathrm{Tq}$

I briefly review the recently proposed construction of the Bethe ansatz which diagonalizes the Hamiltonian for quantum strings on $\mathrm{AdS}_{5} \times \mathrm{S}^{5}$ at large tension and restricted to the large charge states from a closed $\mathfrak{s u}(2)$ subsector.

Copyright line will be provided by the publisher

\section{Introduction}

Recently there has been a lot of effort to shed more light on the AdS/CFT duality conjecture by using the idea of exact integrability. On the gauge theory side this includes elucidation of integrable properties of the dilatation operator of the planar $\mathcal{N}=4 \mathrm{SYM}$ at the leading [1, 2] and higher orders [3] of perturbation theory (see also [4] for earlier account of integrable structures in QCD). On the string side recent developments are related to the study [5] of spinning strings in $\mathrm{AdS}_{5} \times \mathrm{S}^{5}$ which provides new interesting information beyond the plane-wave limit [6]. It appears that underlying integrability of the string sigma model is indispensable for constructing explicit string solutions [7]. Another important aspect concerns the near plane-wave quantization of strings. Here the main problem is to determine corrections to energies of the plane-wave states arising in the large curvature expansion [8] 9].

A relation between gauge and string theories can be probed by comparing the scaling dimensions $\Delta(\lambda)$ of the gauge theory operators ( $\lambda$ is the 't Hooft coupling) with energies $\mathbf{E}(\lambda)$ of the corresponding classi$\mathrm{cal} /$ quantum string configurations. Since the planar dilatation operator admits interpretation as the Hamiltonian of an integrable (long-range) spin chain the powerful method of the algebraic Bethe ansatz can be applied to compute $\Delta(\lambda)$. Recently an all-loop asymptotic Bethe ansatz for the dilatation operator acting in the closed $\mathfrak{s u}(2)$ subsector was proposed [10]. It provides a natural higher-loop generalization of the previously found the one-loop [1], two-loop and three-loop Bethe ansätze [11] compatible with the assumption of the so-called BMN scaling.

Quite generally, integrability implies the existence of a family of local commuting integrals of motion (charges) containing the Hamiltonian [12]. Therefore, comparison of scaling dimensions with string energies can be naturally extended to the whole towers of higher hidden gauge/string charges [13]. The simplest string solutions correspond to rigid strings and they can be described in terms of the finite-dimensional integrable systems of the Neumann type [7]. More general (finite-gap) solutions are encoded in the integral equations of the Bethe type which are referred as the classical string Bethe equations (CSBE) [14].

As is known, the scaling dimensions of operators from the $\mathfrak{s u}(2)$ subsector agree with energies of rigid strings up to two-loop level [15 7, 11] but start to disagree starting at three loops [11]. The same applies to the eigenvalues of all higher commuting charges [13]. Study of the curvature corrections to the plane-wave limit also reveals the similar pattern [9]. One hope for curing this disagreement is to take into account the so-called wrapping interactions [10]. By now, the match/mismatch of the gauge and string integrable

* Corresponding author: e-mail: agleb@aei.mpg.de, Phone: +490331 5677229 Fax: +490331567 7297 
structures can be shown in three different ways. The first one consists in computing the infinite tower of string charges by using the Bäcklund transform on rigid strings and further comparing it with that of the gauge theory [13] (see also [16]). The second is based on matching the CSBE with the gauge theory Bethe ansätze [14]. Finally, the third method uses the so-called ferromagnetic sigma model [17]. A lot of important work on matching the particular gauge/string solutions as well as on understanding the integrable properties of gauge and string theories has been done. Unfortunately, we are not able to discuss it here and refer the reader to the original literature.

The CSBE describing the finite-gap solutions of the string sigma model is an equation of the integral type [14]. On the other hand, the gauge theory asymptotic Bethe ansatz (ABA) is a set of discrete (fundamental) equations. Assuming the validity of the AdS/CFT correspondence one should expect existence of a Bethe ansatz for quantum strings which would serve as a discretization of the integral (continuous) Bethe equations for classical strings and, from the gauge theory perspective, include terms responsible for wrapping interactions. Recently a certain discretization of the CSBE has been proposed [18]. Here we briefly review the essential points of this construction, to which we refer as the quantum string Bethe equation (QSBE). Before we proceed with technical issues let us summarize the properties of the proposed QSBE:

1. In the thermodynamic limit it describes the classical spinning strings.

2. For the finite number of excitations (impurities), $M=2,3$, it reproduces the near plane-wave correction to energies of the quantum string found in [9] and gives a new prediction for any finite $M$.

3. At strong coupling it reproduces the $\sqrt[4]{\lambda}$ behavior of string energies/anomalous dimensions.

4. It agrees with gauge theory asymptotic Bethe ansatz up to two loops.

5. It admits interpretation in terms of a long-range spin chain at weak coupling [19].

The general multi-impurity spectrum (in the $\mathfrak{s u}(2)$ subsector) predicted in [18] has been recently reproduced from the quantized string theory in the near plane-wave background [20]! It would be important to extend the present construction to other closed subsectors (for certain extensions of the CSBE see [21])and ultimately to the whole theory (see [22]). Perhaps, the formidable problem of deriving the QSBE could be approached along the lines of [23]. In [23] we have obtained the classical bosonic Hamiltonian for strings on $\mathrm{AdS}_{5} \times \mathrm{S}^{5}$ in the uniform gauge, showed its integrability and exhibited the corresponding spectral properties. Finally, we note that it would be also interesting to understand the implications of the gauge theory ABA and the QSBE for the Operator Product Expansion in $\mathcal{N}=4$ SYM [24].

\section{Classical String Bethe Equation}

A starting point in our construction of the QSBE is the integral Bethe equation which describes the finitegap solutions of the classical string sigma model. Therefore, it is useful here to recall its origin [14].

Consider a classical string moving in $\mathbb{R} \times \mathrm{S}^{3}$. Here $\mathbb{R}$ stands for the global time direction in $\mathrm{AdS}_{5}$ and $\mathrm{S}^{3}$ is a three-sphere inside $\mathrm{S}^{5}$. The non-vanishing embedding coordinates $X_{1}, \ldots, X_{4}$ are combined in a $\mathrm{SU}(2)$ matrix

$$
g=\left(\begin{array}{cc}
X_{1}+i X_{2} & X_{3}+i X_{4} \\
-X_{3}+i X_{4} & X_{1}-i X_{2}
\end{array}\right) .
$$

This matrix is used to define an $\mathfrak{s u}(2)$-current

$$
A_{\tau}=g^{\dagger} \partial_{\tau} g, \quad A_{\sigma}=g^{\dagger} \partial_{\sigma} g,
$$

because $X_{i}^{2}=1$. The Virasoro constraints read as

$$
\operatorname{Tr}\left(A_{\tau}^{2}+A_{\sigma}^{2}\right)=-\frac{2 \mathbf{E}^{2}}{\lambda}, \quad \operatorname{Tr}\left(A_{\tau} A_{\sigma}\right)=0,
$$


where $\mathbf{E}$ is the space-time energy of the string.

Introduce the $x$-dependent matrices $\mathscr{L}_{\sigma}$ and $\mathscr{L}_{\tau}$ (the Lax connection):

$$
\mathscr{L}_{\sigma}=\frac{z}{1-z^{2}} A_{\tau}+\frac{1}{1-z^{2}} A_{\sigma}, \quad \mathscr{L}_{\tau}=-\frac{1}{1-z^{2}} A_{\tau}-\frac{z}{1-z^{2}} A_{\sigma} .
$$

Here $z \in \mathbb{C}$ is the spectral parameter. One can show that the equations of motion of the sigma model:

$$
\partial_{\tau} A_{\tau}-\partial_{\sigma} A_{\sigma}=0
$$

are equivalent to the condition of zero curvature [12]

$$
\left[\mathscr{D}_{\tau}, \mathscr{D}_{\sigma}\right]=0
$$

for covariant derivatives $\mathscr{D}_{\tau}=\partial_{\tau}-\mathscr{L}_{\tau}$ and $\mathscr{D}_{\sigma}=\partial_{\sigma}-\mathscr{L}_{\sigma}$.

The Lax connection can be used to construct an infinite tower of integrals of motion for the evolution equations (11). Define the monodromy matrix as the path-ordered exponential of $\mathscr{L}_{\sigma}$ :

$$
\mathscr{T}(z)=\mathscr{P} \exp \int_{0}^{2 \pi} \mathscr{L}_{\sigma}(z) \mathrm{d} \sigma .
$$

Using the condition of zero curvature one can easily show that the spectral invariants of the monodromy matrix are conserved under the time evolution. Let us parametrize the eigenvalues of $\mathscr{T}(z)$ in the following way

$$
\mathscr{T}(z)=\left(\begin{array}{ll}
e^{i p(z)} & \\
& e^{-i p(z)}
\end{array}\right)
$$

The function $p(z)$ is usually referred as quasi-momentum. The basic idea of the finite-gap integration is to reconstruct the quasi-momentum and, more generally, solutions of the evolution equations by using the analyticity properties of $p(z)$. The quasi-momentum has at least the same two first order poles at $z= \pm 1$ as the Lax connection itself. The residues are $\pm \frac{\pi \mathbf{E}}{\sqrt{\lambda}}$ (the dependence on $\mathbf{E}$ occurs due to the Virasoro constraints); they can be easily determined (up to the sign ambiguity!) by diagonalizing the pole part of $\mathscr{L}_{\sigma}$. We further assume that $p(z)$ has no other poles. This allows one to define a resolvent

$$
\mathbf{G}(z) \equiv p(z)+\frac{\mathbf{E}}{\sqrt{\lambda}} \frac{\pi}{z-1}+\frac{\mathbf{E}}{\sqrt{\lambda}} \frac{\pi}{z+1},
$$

which is a pole-free analytic function on the complex $z$-plane with a (finite) number of cuts. One can show that the asymptotics of $p(z)$ around $z=0$ and $z=\infty$ are related to the Noether charges of the global symmetry group $\mathrm{SU}(2)_{\mathrm{L}} \times \mathrm{SU}(2)_{\mathrm{R}}$ which, in their turn, can be expressed via the Dynkin labels of the corresponding representations. Below we just state the asymptotic properties of $\mathbf{G}(z)$ referring the reader to the original work [14] for further details:

$$
\begin{array}{ll}
\mathbf{G}(z) \rightarrow \frac{2 \pi(\mathbf{E}-L+2 M)}{z \sqrt{\lambda}}+\ldots, & \text { when } \quad x \rightarrow \infty, \\
\mathbf{G}(z) \rightarrow 2 \pi m+z \frac{2 \pi(\mathbf{E}-L)}{\sqrt{\lambda}}+\ldots, & \text { when } \quad x \rightarrow 0 .
\end{array}
$$

Here $m \in \mathbb{Z}$ is a winding number.

The analytic function $\mathbf{G}(z)$ can be expressed via the spectral density $\rho_{\mathrm{s}}(z)$ as

$$
\mathbf{G}(z)=\int_{\mathbf{C}} \frac{\mathrm{d} z^{\prime} \rho_{\mathbf{s}}\left(z^{\prime}\right)}{z-z^{\prime}} .
$$


The spectral density is supported an a finite number of cuts $\mathbf{C}$.

Finally, unimodularity of the monodromy matrix implies that on every cut one has

$$
p(z+i 0)+p(z-i 0)=2 \pi n, \quad n \in \mathbb{Z} .
$$

Since

$$
\mathbf{G}(z+i 0)+\mathbf{G}(z-i 0)=\int_{\mathbf{C}}\left(\frac{\rho_{\mathrm{s}}(z)}{z-z^{\prime}+i 0}+\frac{\rho_{\mathrm{s}}(z)}{z-z^{\prime}-i 0}\right)=2 \int_{\mathbf{C}} \frac{\rho_{\mathrm{s}}\left(z^{\prime}\right)}{z-z^{\prime}} .
$$

one gets (for every cut) the integral equation of the Bethe type

$$
f_{\mathbf{C}} \frac{\rho_{\mathrm{s}}\left(z^{\prime}\right)}{z-z^{\prime}}=\frac{\mathbf{E}}{L} \frac{\pi z}{z^{2}-1}+\pi n,
$$

which is the CSBE [14].

The final step consists in changing the spectral parameter $z \rightarrow z(\varphi)$ according to the rule [10]

$$
z \rightarrow z(\varphi)=\frac{\varphi+\sqrt{\varphi^{2}-4 \omega^{2}}}{2 \omega}, \quad \omega^{2} \equiv \frac{\lambda}{16 \pi^{2} L^{2}} .
$$

This change of variables leads to the standard (field-theoretic type) normalization of the density

$$
\int_{\mathbf{C}} d \varphi \rho_{\mathrm{s}}(\varphi)=\frac{M}{L}=\alpha,
$$

while the CSBE acquires rather complicated form [10]

$$
\begin{aligned}
& f_{\mathbf{C}} \frac{d \varphi^{\prime} \rho_{\mathrm{s}}\left(\varphi^{\prime}\right)}{\varphi-\varphi^{\prime}}=\frac{1}{2 \sqrt{\varphi^{2}-4 \omega^{2}}}+\pi n+ \\
& +\omega^{2} \int_{\mathbf{C}} \frac{d \varphi^{\prime} \rho_{\mathrm{s}}\left(\varphi^{\prime}\right)}{\sqrt{\varphi^{2}-4 \omega^{2}} \sqrt{\varphi^{\prime 2}-4 \omega^{2}}} \frac{\varphi-\sqrt{\varphi^{2}-4 \omega^{2}}-\varphi^{\prime}+\sqrt{\varphi^{\prime 2}-4 \omega^{2}}}{\left(\varphi+\sqrt{\varphi^{2}-4 \omega^{2}}\right)\left(\varphi^{\prime}+\sqrt{\varphi^{\prime 2}-4 \omega^{2}}\right)-4 \omega^{2}} .
\end{aligned}
$$

\section{Quantum String Bethe Equation}

Let us assume that there exists a set of fundamental (discrete) equations of the Bethe type which in the thermodynamic (continuum) limit leads to the integral equation (3). In general, the procedure of discretization is by no means unique. We show, however, that there exists a distinguished set of fundamental equations, which agrees with all our current knowledge about quantum strings on $\mathrm{AdS}_{5} \times \mathrm{S}^{5}$.

A crucial observation consists in rewriting eq. (3) in the following form [18]

$$
f_{\mathbf{C}} \frac{d \varphi^{\prime} \rho_{\mathrm{s}}\left(\varphi^{\prime}\right)}{\varphi-\varphi^{\prime}}=\pi n+\frac{1}{2} \mathbf{q}_{1}(\varphi)+\sum_{r=0}^{\infty} \omega^{2 r+4} \mathbf{q}_{[r+3}(\varphi) \mathbf{Q}_{r+2]} .
$$

Here the bracket $[.,$.$] stands to denote antisymmetrization of indices and we use the notation$

$$
\mathbf{Q}_{r}=\int_{\mathbf{C}} d \varphi \rho_{\mathrm{s}}(\varphi) \mathbf{q}_{r}(\varphi), \quad \mathbf{q}_{r}(\varphi)=\frac{1}{\sqrt{\varphi^{2}-4 \omega^{2}}} \frac{1}{\left(\frac{1}{2} \varphi+\frac{1}{2} \sqrt{\varphi^{2}-4 \omega^{2}}\right)^{r-1}}
$$

Now it is time to realize that the charges $\mathbf{q}_{r}(\varphi)$ are precisely those which arise in the thermodynamic limit ( $M, L \rightarrow \infty, M / L=\alpha$ fixed) from the local excitation charges of the gauge theory ABA [10]. In the momentum basis these charges are [10]

$$
\mathbf{q}_{r}(p)=\frac{2 \sin \left(\frac{r-1}{2} p\right)}{r-1}\left(\frac{\sqrt{1+8 g^{2} \sin ^{2}\left(\frac{1}{2} p\right)}-1}{2 g^{2} \sin \left(\frac{1}{2} p\right)}\right)^{r-1}, \quad g^{2}=\frac{\lambda}{8 \pi^{2}} .
$$


In particular,

$$
\begin{aligned}
& \mathbf{q}_{1}(p)=p \Leftarrow \text { Momentum } \\
& \mathbf{q}_{2}(p)=\frac{1}{g^{2}}\left(\sqrt{1+8 g^{2} \sin ^{2}\left(\frac{1}{2} p\right)}-1\right) \Leftarrow \text { Energy } .
\end{aligned}
$$

To rewrite this charges in terms of the variable $\varphi$ one has to invert the phase function of ABA [10]

$$
\varphi(p)=\frac{1}{2} \cot \left(\frac{1}{2} p\right) \sqrt{1+8 g^{2} \sin ^{2}\left(\frac{1}{2} p\right)} .
$$

Such a remarkable connection between classical string and quantum gauge theory emerging in the thermodynamic limit can be further used to infer a possible fundamental form of the string Bethe equations.

The Bethe equations we propose to describe the leading quantum effects for strings in the $\mathfrak{s u}(2)$ sector are of the form [18]

$$
\exp \left(i L p_{k}\right)=\prod_{\substack{j=1 \\ j \neq k}}^{M} \mathrm{~S}\left(p_{k}, p_{j}\right), \quad \sum_{k=1}^{M} p_{k}=0
$$

Here $\mathrm{S}\left(p_{k}, p_{j}\right)$ is the $\mathrm{S}$-matrix for pairwise scattering of local excitations with momenta $p_{k}$ :

$$
\mathrm{S}\left(p_{k}, p_{j}\right)=\underbrace{\frac{\varphi\left(p_{k}\right)-\varphi\left(p_{j}\right)+i}{\varphi\left(p_{k}\right)-\varphi\left(p_{j}\right)-i}}_{\text {S-matrix of ABA }} \exp \left(2 i \sum_{r=0}^{\infty}\left(\frac{g^{2}}{2}\right)^{r+2} \mathbf{q}_{r+2}\left(p_{[k}\right) \mathbf{q}_{r+3}\left(p_{j]}\right)\right) .
$$

The string S-matrix is a product of two terms. The first term involves the phase function $\varphi(p)$ and it is identical to the S-matrix of the gauge theory ABA constructed in [10]. The second exponential term is built upon the local excitation charges eq. (4) of gauge theory. It is precisely this term which makes a difference between gauge and string theory! It starts to contribute to the S-matrix at order $\lambda^{2}$ which, from the point of view of gauge theory, means the three-loop level of perturbation theory.

One should view eqs. (5) as the system of equations to determine $M$ individual momenta $p_{k}$. As momenta are found they can be further used to compute the string energy

$$
\mathbf{E}(g)=L+g^{2} \sum_{k=1}^{M} \mathbf{q}_{2}\left(p_{k}\right)
$$

as well as the higher charges, $\mathbf{Q}_{r}=\sum_{k=1}^{M} \mathbf{q}_{r}\left(p_{k}\right)$. Finally, we note that taking the logarithms of eqs.(5) and passing through the standard rooting of performing the thermodynamic limit we indeed recover the integral equation (3).

The properties of the QSBE have been already listed in the Introduction and their derivation can be found in [18]. Here we note that the QSBE can be easily solved in the $1 / J$ expansion, where $J=L-M$. Up to the order $1 / J$ the string energy is

$$
\mathbf{E}=J+\sum_{k=1}^{M} \sqrt{1+\lambda^{\prime} n_{k}^{2}}-\frac{\lambda^{\prime}}{J} \sum_{\substack{k, j=1 \\ j \neq k}}^{M} \frac{n_{k}}{n_{k}-n_{j}}\left(n_{j}^{2}+n_{k}^{2} \sqrt{\frac{1+\lambda^{\prime} n_{j}^{2}}{1+\lambda^{\prime} n_{k}^{2}}}\right),
$$

where $n_{k} \neq n_{j}$ are the mode numbers of local excitations. This formula beautifully agrees with the nearplane wave spectrum of the quantized string theory [20]. The same remains true also for the case of the confluent mode numbers, see [18] for details. 


\section{Acknowledgments}

I would like to thank Sergey Frolov and Matthias Staudacher for enjoyable collaboration on the subject presented here. I'm grateful to Niklas Beisert and Arkady Tseytlin for many interesting discussions. I also thank the organizers and the participants of the RTN-EXT Workshop in Kolymbari for the stimulating atmosphere and interesting discussions. The work was supported in part by the European Commission RTN programme HPRN-CT-2000-00131 and by RFBI grant N02-01-00695.

\section{References}

[1] J.A. Minahan and K. Zarembo, JHEP 0303, 013 (2003), hep-th/0212208

[2] N. Beisert, Nucl. Phys. B 676, 3 (2004), hep-th/0307015

N. Beisert and M. Staudacher, Nucl. Phys. B 670, 439 (2003), hep-th/0307042

[3] N. Beisert, C. Kristjansen and M. Staudacher, Nucl. Phys. B 664, 131 (2003), hep-th/0303060

[4] L.N. Lipatov, JETP Lett. 59, 596 (1994), hep-th/9311037

L.D. Faddeev and G.P. Korchemsky, Phys. Lett. B 342, 311 (1995), hep-th/9404173

V.M. Braun, S.E. Derkachov, G.P. Korchemsky and A.N. Manashov, Nucl. Phys. B 553, 355 (1999).

[5] S. Frolov and A.A. Tseytlin, JHEP 0206, 007 (2002), hep-th/0204226

S. Frolov and A.A. Tseytlin, Nucl. Phys. B 668, 77 (2003), hep-th/0304255

S. Frolov and A.A. Tseytlin, Phys. Lett. B 570, 96 (2003), hep-th/0306143

[6] D. Berenstein, J.M. Maldacena and H. Nastase, JHEP 0204, 013 (2002), hep-th/0202021

[7] G. Arutyunov, S. Frolov, J. Russo and A. A. Tseytlin, Nucl. Phys. B 671, 3 (2003), hep-th/0307191

G. Arutyunov, J. Russo and A. A. Tseytlin, Phys. Rev. D 69, 086009 (2004), hep-th/0311004

[8] A. Parnachev and A. V. Ryzhov, JHEP 0210, 066 (2002), hep-th/0208010

[9] C. G. Callan, H. K. Lee, T. McLoughlin, J. H. Schwarz, I. Swanson and X. Wu, Nucl. Phys. B 673, 3 (2003).

C. G. Callan, T. McLoughlin and I. Swanson, hep-th/0404007

C. G. Callan, T. McLoughlin and I. Swanson, hep-th/0405153

[10] N. Beisert, V. Dippel and M. Staudacher, JHEP 0407, 075 (2004), hep-th/0405001

[11] D. Serban and M. Staudacher, JHEP 0406, 001 (2004), hep-th/0401057

[12] L.D. Faddeev and L.A. Takhtajan, Hamiltonian Methods in the Theory of Solitons (Springer, 1987).

L.D. Faddeev, Integrable Models in $(1+1)$-Dimensional Quantum Field Theory in: Recent Advances in Field Theory and Statistical Mechanics, Proceedings of the Les Houches Summer School 1982, North-Holland: Amsterdam 1984, pp. 561-608.

L. Faddeev, hep-th/9605187

[13] G. Arutyunov and M. Staudacher, JHEP 0403, 004 (2004), hep-th/0310182

G. Arutyunov and M. Staudacher, hep-th/0403077

[14] V. A. Kazakov, A. Marshakov, J. A. Minahan and K. Zarembo, JHEP 0405, 024 (2004), hep-th/0402207

[15] N. Beisert, J. A. Minahan, M. Staudacher and K. Zarembo, JHEP 0309 (2003) 010, hep-th/0306139

N. Beisert, S. Frolov, M. Staudacher and A. A. Tseytlin, JHEP 0310, 037 (2003), hep-th/0308117

J. Engquist, J. A. Minahan and K. Zarembo, JHEP 0311, 063 (2003), hep-th/0310188

[16] J. Engquist, JHEP 0404, 002 (2004), hep-th/0402092

[17] M. Kruczenski, hep-th/0311203

M. Kruczenski, A. V. Ryzhov and A. A. Tseytlin, hep-th/0403120

[18] G. Arutyunov, S. Frolov and M. Staudacher, JHEP 0410, 016 (2004), hep-th/0406256

[19] N. Beisert, hep-th/0409054

[20] T. McLoughlin and I. Swanson, hep-th/0407240

[21] V. A. Kazakov and K. Zarembo, hep-th/0410105

N. Beisert, V. A. Kazakov and K. Sakai, hep-th/0410253

[22] M. Staudacher, to appear.

[23] G. Arutyunov and S. Frolov, hep-th/0411089

[24] G. Arutyunov, B. Eden, A. C. Petkou and E. Sokatchev, Nucl. Phys. B 620, 380 (2002), hep-th/0103230 G. Arutyunov, S. Frolov and A. Petkou, Nucl. Phys. B 602, 238 (2001), hep-th/0010137 